\title{
Teste de Voo como Critério de Avaliação da Qualidade de Palmistichus elaeisis Delvare \& LaSalle (Hymenoptera: Eulophidae)
}

\author{
Elidiane Feltrin-Campos ${ }^{\bowtie}$, Priscila Laranjeira Rôdas¹, Daniele Fabiana Glaeser², \\ Fabrício Fagundes Pereira ${ }^{3}$ \& Harley Nonato de Oliveira ${ }^{2}$
}

1. Pós Graduação em Entomologia e Conservação da Biodiversidade, Universidade Federal da Grande Dourados, e-mail: elidifeltrin@hotmail.com (Autor para correspondência ${ }^{\varpi}$ ), prih davis@hotmail.com. $\quad$ 2. Embrapa Agropecuária Oeste, e-mail: daniglaeser@yahoo.com.br, harley.oliveira@embrapa.br. 3. Universidade Federal da Grande Dourados/Faculdade de Ciências Biológicas e Ambientais, e-mail: fabriciofagundes@ufgd.edu.br.

\section{EntomoBrasilis 9 (2): 137-139 (2016)}

Resumo. A produção de inimigos naturais com qualidade comparável àqueles encontrados na natureza é primordial para os sistemas de criação de insetos em condições de laboratório, que vise atender a programas de controle biológico. Dentre os indicadores de qualidade de parasitoides, a capacidade de voo é uma característica importante a ser avaliada em laboratório, pois está relacionada à dispersão e alcance de abrigo, alimentos e hospedeiros em condições de campo. Palmistichus elaeisis Delvare \& LaSalle é um endoparasitoide gregário, polífago, idiobionte que parasita pupas, principalmente da ordem Lepidoptera. O objetivo deste trabalho foi avaliar a qualidade da população do parasitoide $P$. elaeisis, criada em pupas Diatraea saccharalis (Fabricius) em laboratório pelo teste de voo. Pupas de D. saccharalis com 24 horas de idade foram expostas ao parasitismo por fêmeas de $P$. elaeisis. Os adultos emergidos foram classificados de acordo com suas posições no interior da Unidade de teste em: voadores, caminhadores e não voadores. A porcentagem dos parasitoides encontrados na tampa (voadores) foi de 51,23\%, no anel (caminhadores) de $23,49 \%$ e no fundo (não voadores) de 25,26\%. Em razão aos percentuais de não voadores observado para outras espécies de parasitoides, o valor constatado para $P$. elaeisis pode significar uma queda na qualidade desse parasitoide, o que levaria a necessidade de introdução de novos indivíduos coletados em campo para recompor essa qualidade de voo.

Palavras-Chave: Controle Biológico; Laboratório; Inimigos Naturais; Parasitoide; Praga.

\section{Flight Test as Evaluation Criteria of the Palmistichus elaeisis Delvare \& LaSalle (Hymenoptera: Eulophidae) Quality}

Abstract. The production of natural enemies of comparable quality to those found in nature is essential to the creation of systems of insect in laboratory conditions, which object to meet biological control programs. Among the indicators of quality of parasitoids, flight capacity is an important factor to be evaluated in the laboratory feature, because it is related to the dispersion and range of shelter, food and hosts under field conditions. Palmistichus elaeisis Delvare \& LaSalle is a gregarious endoparasitoid, polyphagous, idiobionte and parasite pupae, especially the Lepidoptera order. The objective of this study was to evaluate the quality of the parasitoid P. elaeisis population, created in pupae of Diatraea saccharalis (Fabricius) laboratory for the flight test. Pupae $D$. saccharalis with 24 hours of age were exposed to parasitism by females of $P$. elaeisis. The emerged adults were classified according to their positions within the unit test, flyers, running and not flying. The percentage of parasitoids found on the cover (flying) was $51.23 \%$, the ring (running) of $23.49 \%$ and the bottom (non-flying) of $25.26 \%$. Because the percentage of non-flying observed for other species of parasitoids, the value found for $P$. elaeisis could mean a drop in quality of the parasitoid, which would lead to the need to introduce new individuals collected in the field to restore this quality of flight.

Keywords: Biological Control; Laboratory; Natural Enemies; Parasitoid; Pest.

Pp almistichus elaeisis Delvare \& LaSalle é um endoparasitoide gregário, polífago, idiobionte que parasita pupas de Lepidoptera (PEREIRA et al. 2008), tais como Eupseudosoma involuta (Sepp) (Arctiidae), Euselasia eucerus Hewitson (Riodinidae), Anticarsia gemmatalis (Hübner) (Noctuidae), Diatraea saccharalis (Fabricius) (Crambidae), Heliothis virescens (Fabricius) (Noctuidae) e Spodoptera frugiperda (J.E. Smith) (Noctuidae) (BITTENCOURT \& Berti Filho 1999). Esse parasitoide vem sendo estudado como um importante agente no controle de insetos-praga florestais e agrícolas (PEREIRA et al. 2009) e por isso, vem sendo criado em laboratório de algumas instituições de Ensino e Pesquisa para possíveis liberações em campo.

A produção de inimigos naturais com qualidade comparável àqueles encontrados na natureza é um dos principais objetivos de qualquer laboratório de criação que vise atender às exigências dos programas de controle biológico. Dessa forma, para determinar o sucesso das criações massais, teste de controle de qualidade são realizados para assegurar que um inimigo natural multiplicado em laboratório seja eficiente no controle de pragas quando liberados a campo (Рвеzotті et al. 2002).

Uma das características avaliadas como controle de qualidade é a capacidade de voo e de caminhar dos inimigos naturais, pois estas estão envolvidas com a dispersão e forrageamento em campo (Gardner \& Lenteren 1986; PrezotTi et al. 2002). Essas características podem variar durante a criação massal, principalmente se os parasitoides são criados por várias gerações sucessivas, e por isso, devem ser monitoradas para a eficiência no controle de pragas (РRеzotті et al. 2002).

O objetivo deste trabalho foi avaliar a qualidade da população do parasitoide $P$. elaeisis, criada em pupas de $D$. saccharalis em laboratório pelo teste de voo. 
Como critério de avaliação utilizou-se o teste de voo, modelo ESALQ com adaptações. Para o teste utilizou-se cilindro de PVC com o interior recoberto por cartolina preta. O fundo do tubo de PVC foi vedado com plástico preto ajustado com disco de isopor. Para determinar os parasitoides caminhadores, na parte interna do tudo de PVC, um anel de cola foi pincelado a $3,5 \mathrm{~cm}$ da extremidade inferior do tubo. Uma placa de Petri transparente contendo cola entomológica foi encaixada na parte superior do cilindro, como armadilha para os parasitoides em voo. Cinco pupas de $D$. saccharalis foram individualizadas em tubos de vidro e expostas ao parasitismo por cinco fêmeas de $P$. elaeisis, durante $24 \mathrm{~h}$. Os tubos contendo as pupas parasitadas, prestes à emergência, foram fixados individualmente no centro das unidades-teste e foram colocados sobre uma bancada, abaixo da fonte de luz, onde permaneceram por $72 \mathrm{~h}$. Posteriormente avaliou-se a porcentagem de parasitoides encontrados na tampa de vidro transparente (placa de Petri com $14 \mathrm{~cm}$ de diâmetro x 2 $\mathrm{cm}$ de altura) (voadores), no fundo (não voadores) e no anel de cola (caminhadores) da unidade teste.

O delineamento experimental foi inteiramente casualizado sendo os dados comparados pelo teste de Tukey a 5\% de significância, utilizando-se o programa estatístico Assistat, beta 7.6 (AssisTAT 2011).

A porcentagem média de parasitoides $P$. elaeisis capturados na tampa (voadores), foi de $51,23 \%$, sendo maior que a porcentagem de parasitoides capturados no anel (caminhadores) e no fundo da unidade-teste (não voadores) (Tabela 1).

Tabela 1. Modelo ESALQ para avaliação da atividade de voo de populações de Palmistichus elaeisis, em laboratório, com base na porcentagem de indivíduos capturados em diferentes locais das unidades teste $\left(25 \pm 1^{\circ} \mathrm{C}\right.$, $60 \pm 10 \%$ UR e fotofase de $24 \mathrm{~h}$ ).

\begin{tabular}{cc}
\hline Parasitoides & $\begin{array}{c}\text { Porcentagem de indivíduos capturados dentro } \\
\text { das unidades-teste (\%) }\end{array}$ \\
\hline Voadores (tampa) & $51,23 \mathrm{a}$ \\
Caminhadores (anel) & $23,46 \mathrm{~b}$ \\
Não voadores (fundo) & $25,26 \mathrm{~b}$ \\
\hline CV (\%) & $\mathbf{2 0 , 8 2}$ \\
\hline
\end{tabular}

Médias seguidas da mesma letra na coluna, não diferem pelo teste de Tukey à $5 \%$ de siginificância $(\mathrm{p}<0,05) . \mathrm{CV}=$ Coeficiente de variação.

A soma dos percentuais de voadores e caminhadores foi de 74,7\%, e segundo GARDNER \& LENTEREN (1986) tanto a capacidade de voar quanto de caminhar são características importantes para o desempenho do inimigo natural em condições de campo, pois estão relacionadas com o forrageamento e a dispersão no campo.

O percentual de voadores observados para $P$. elaeisis nesse experimento foram menores do que aqueles obtidos para Trichospilus diatraeae Cherian \& Margabandhu 1942 (Hymenoptera: Eulophidae), quando BELLON et al. (2014) avaliaram o efeito das densidades três, cinco e 14 fêmeas do parasitoide por pupa de $D$. saccharalis sobre a capacidade de voo, com percentuais de 79,08\%; 74,12\% e 64,18\% para cada densidade. A percentagem de $P$. elaeisis voadores também foi menor do que os percentuais observados para Trichogramma pretiosum Riley (Hymenoptera: Trichogrammatidae) (86\%) (PrezotTi et al. 2002), Trichogramma atopovirilia Oatman e Platner (maior que 75\%) (SOARES et al. 2012) e Trichogramma brassicae Bezdenko (61,4\%) (DutTon \& Bigler 1995).

Ressalta-se que quanto maior for o percentual de voadores, melhor é a qualidade do parasitoide no que se refere à capacidade de voo e, portanto, maiores são as chances de sucesso para o controle biológico, já que facilita a dispersão em campo, assim como a capacidade forrageamento e a localização do hospedeiro (PREZotTi et al. 2002).
Apesar do tubo de ensaio utilizado no Modelo ESALQ permitir que os parasitoides após a emergência, percorressem o tubo, tendo tempo para distender suas asas e voar até a tampa da unidadeteste (PrezotTi et al. 2002), muitos parasitoides não foram capturados na tampa, correspondendo aos insetos classificados como caminhadores e não-voadores.

O número de parasitoides capturados nos anéis de cola (caminhadores) foi de $23,46 \%$ (Tabela 1). Esses valores são maiores que aqueles relatados por Rodrigues et al. (2009) e SOAREs et al. (2007) para T. pretiosum, que foram de 11 e $13 \%$ respectivamente. De acordo com PrezotTi et al. (2002) quanto menor o número de indivíduos presos no anel de cola, melhor a qualidade do teste, com maior confiabilidade da avaliação.

A porcentagem de insetos encontrados no fundo (não voadores) das unidades de teste foi de $25,26 \%$ (Tabela 1). Esse percentual foi maior que os valores encontrados para as gerações 35 (7,9\%) e 72 (10\%) de $T$. pretiosum (PrezotTi et al. 2002) e para as diferentes densidade de fêmeas de $T$. diatraeae (três, cinco e 14 fêmeas) por pupa de $D$. saccharalis que foi de $13,9 \%, 7,1 \%$ e 9,9\%, respectivamente (BELLon et al. 2014). Para PREzotTi et al. (2002), a utilização do modelo ESALQ, permite melhor discriminação entre os insetos voadores e não voadores, pois os parasitoides que ao chegarem ao topo do tubo de ensaio, no interior da unidadeteste, ao caminharem em direção ao contrário da luz, indo em direção ao fundo, certamente possui reduzida propensão ao voo ou encontra-se com as asas deformadas.

Em razão aos percentuais de não voadores observado para outras espécies de parasitoides, o valor constatado para $P$. elaeisis pode significar uma queda na qualidade de voo desse parasitoide, o que levaria a necessidade de introdução de novos indivíduos coletados em campo para recompor essa qualidade em condições de laboratório.

\section{REFERÊNCIAS}

Assistat, 2011. Campina Grande: Universidade Federal de Campina Grande. Versão beta 7.6, Disponível em: <http://www.assistat.com/indexp.html >.

Bellon, P.P., H.N. Oliveira \& F.F. Pereira, 2014. Teste de voo como critério de avaliação da qualidade de Trichospilus diatraeae (Hymenoptera: Eulophidae). Bioscience Journal, 30: 582584. Disponível em: <http://www.seer.ufu.br/index.php/ biosciencejournal/article/viewFile/15033/13778>. [Acesso em: 29.vi.2016].

Bittencourt, M.A.L. \& E. Berti Filho, 1999. Preferência de Palmistichus elaeisis por pupas de diferentes lepidópteros pragas. Scientia Agricola, 56: 1281-1283. DOI: http://dx.doi. org/10.1590/So103-90161999000500033.

Dutton, A. \& F. Bigler, 1995. Flight activity assessment of the egg parasitoid Trichogramma brassicae (Hymenoptera: Trichogrammatidae) in laboratory and field conditions. Entomophaga, 40: 223-233. DOI: http://dx.doi.org/10.1007/ bfo2373070.

Gardner, S.M. \& J.C. Lenteren, 1986. Characterization of the arrestment responses of Trichogramma evanescens. Oecologia, 68: 265-270. DOI: http://dx.doi.org/10.1007/ BFo0384798.

Pereira, F.F., J.C. Zanuncio, J.E. Serrão, P.L. Pastori \& F.S. Ramalho, 2009. Reproductive performance of Palmistichus elaeisis Delvare and LaSalle (Hymenoptera: Eulophidae) with previously refrigerated pupae of Bombyx mori L. (Lepidoptera: Bombycidae). Brazilian Journal of Biology, 69: 865-869. DOI: http://dx.doi.org/10.1590/S151969842009000400014 .

Pereira, F.F., T.V. Zanuncio, J.C. Zanuncio, D. Pratissoli \& M.T. Tavares, 2008. Species of Lepidoptera defoliators of eucalyptus as new host for the parasitoid Palmistichus elaeisis (Hymenoptera: Eulophidae). Brazilian 
Archives of Biology and Technology, 51: 259-262. DOI: http://dx.doi.org/10.1590/S1516-89132008000200004.

Prezotti, L., J.R.P. Parra, R. Vencovsky, C.T. Dias, I. Cruz \& M.C.M. Chagas, 2002. Teste de voo como critério de avaliação da qualidade de Trichogramma pretiosum Riley (Hymenoptera: Trichogrammatidae). Adaptação de metodologia. Neotropical Entomology, 31: 411-417. DOI: http://dx.doi.org/10.1590/ S1519-566X2002000300010.

Rodrigues, S.M.M., M.V. Sampaio \& J.E. Miranda, 2009. Avaliação da capacidade de voo, parasitismo e emergência de linhagens de Trichogramma pretiosum Riley (Hymenoptera: Trichogrammatidae). Arquivos do Instituto Biológico, 76: 749-753.

Soares, M.A., G.L.D. Leite, J.C.Zanuncio, S.L. Rocha, V.G.M.deSá, \& J.E. Serrão, 2007. Flight capacity, parasitism and emergence of five Trichogramma (Hymenoptera: Trichogrammatidae) species from Forest areas in Brazil. Phytoparasitica, 35: 314318. DOI: http://dx.doi.org/10.1007/BFo2981165.
Soares, M.A., G.L.D. Leite, J.C. Zanuncio, V.G.M. de Sá, C.S. Ferreira, S.L. Rocha, M. Pires \& J.E. Serrão, 2012. Quality Control of Trichogramma atopovirilia and Trichogramma pretiosum (Hym.: Trichogrammatidae) Adults Reared Under Laboratory Conditions. Brazilian Archives Biology Technology, 55: 305-311. DOI: http://dx.doi.org/10.1590/ S1516-89132012000200018.

\section{Recebido em: 24.xi.2015}

Aceito em: 26.vi.2016

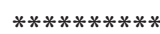

\section{Como citar este artigo:}

Feltrin-Campos, E., P.L. Rôdas, D.F. Glaeser, F.F. Pereira \& H.N. Oliveira, 2016. Teste de Voo como Critério de Avaliação da Qualidade de Palmistichus elaeisis Delvare \& LaSalle (Hymenoptera: Eulophidae). EntomoBrasilis, 9 (2): 137-139.

Acessível em: doi:10.12741/ebrasilis.vgi2.571
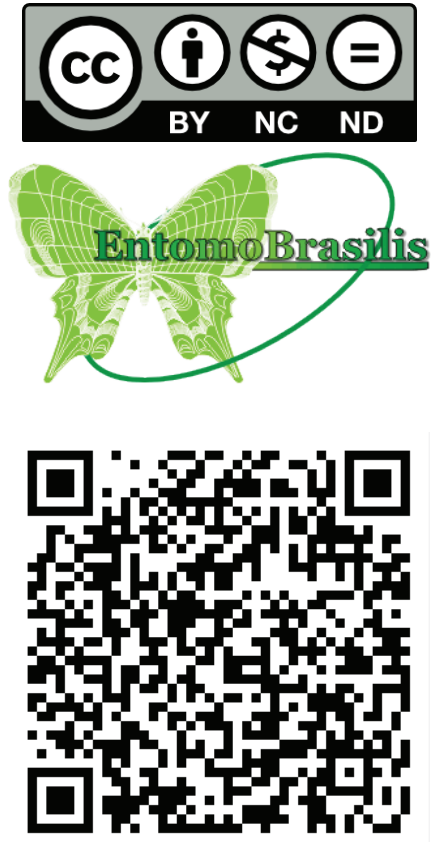